\title{
Jitter Correction and SNR Improvement of A-Scan Signal Using Wavelet Denoising and Signal Averaging for a Portable Time-Domain Optical Coherence Tomography System
}

\author{
Maria Cecilia D. Galvez ${ }^{\mathrm{a}, \mathrm{b}, *}$, Edgar A. Vallar ${ }^{\mathrm{a}, \mathrm{b}}$, Tatsuo Shiina ${ }^{\mathrm{c}}$, Ernest P. Macalalad ${ }^{\mathrm{d}}$, Paulito F. Mandia ${ }^{\mathrm{a}, \mathrm{b}}$ \\ ${ }^{a}$ Environment and RemoTe Sensing ResearcH (EARTH) Laboratory, Physics Department, De La Salle University, \\ Malate, Manila, Philippines \\ ${ }^{b}$ Applied Research for Community, Health and Environment Resilience and Sustainability (ARCHERS), Center for Natural Sciences and \\ Environmental Research (CENSER), De La Salle University, Malate, Manila, Philippines \\ ${ }^{c}$ Graduate School of Science and Engineering, Chiba University, Inage-ku, Chiba, Japan \\ ${ }^{d}$ Department of Physics, Mapúa University, 658 Muralla St., Intramuros, Manila, Philippines \\ Corresponding author: " maria.cecilia.galvez@dlsu.edu.ph
}

\begin{abstract}
Optical coherence tomography (OCT) is used to probe surface cross-sections of various materials in manufacturing, anatomy, and agriculture, among others. In any OCT system, signals have embedded noise in various forms, such as electrical noise and Jitter, which affect the depth profile (A-scan) and image quality. A signal processing method for correcting the Jitter and improving the signalto-noise ratios (SNR) and image quality was developed in this study for a portable time-domain (TD) OCT system that utilizes a pcbased oscilloscope for data acquisition. A stack of five glass coverslips was used as a sample. Each signal from the oscilloscope consists of an A-scan coupled with a trigger signal. Jitter correction was done by first denoising the trigger signal using a combination of moving average and wavelet denoising. A reference trigger was selected, and all other trigger signals were adjusted, including the A-scans. Once jitter was corrected, the denoising method on the OCT A-scans was employed. The signal averaging method and various wavelet denoising methods were applied to the A-scans of the sample to identify which will give the highest SNR and improved image quality. Combining averaging of fifty signals and Daubechies 7 (Db7) with hard thresholding reduced the acquisition time and storage space by $50 \%$, improved the SNR by $18 \mathrm{~dB}$, improved the depth profile and image quality of a stack of five glass coverslips. This signal processing method will allow us to characterize and properly visualize cross-sectional images of other samples in the future using our TD-OCT system.
\end{abstract}

Keywords - A-scan; denoising; optical coherence tomography; signal-to-noise ratio; wavelets.

\section{INTRODUCTION}

Optical Coherence Tomography (OCT) started in 1992 as an imaging modality for the retina [1], which subsequently expanded to image other parts of the body such as skin,[2] [3] and dental tissue, [4]. Later on, it has been used in various fields such as manufacturing [5] and agriculture [6]. This popularity is because of the non-destructive nature of OCT, i.e., a cross-sectional image can be obtained without the need for the sample to be dissected or broken apart. The basic principle of OCT originated from the Michelson interferometer. An OCT system technology can be a timedomain OCT (TD-OCT) and frequency-domain OCT (FD-
OCT). The main difference between the two is that the former uses a scanning mechanism to modulate the optical length of the reference path continuously and the intensity of the interference signal is maximum when the sample and reference path are equal, whereas the latter has a fixed mirror that generate images by analyzing the spectral pattern of the collected interferograms generated by combining the reflected lights from the reference and the sample arms [7].

This study used a portable version of TD-OCT, which uses a rotating retroreflector [8] instead of the traditionally used galvanometer actuated mirror [9] as part of the reference arm. The setup is connected to a pc-based oscilloscope. One channel is attached to a photo-interrupter that generates a signal that serves as the oscilloscope's trigger source. Another 
channel is attached to the OCT signal processing circuit board to produce an A-scan, which is the main OCT signal because it carries the depth profile of the sample under study. When these A-scans are combined, it produces a two-dimensional image (B-scan). However, the image quality and penetration depth of any OCT system is affected by noise. In the case of our portable TD-OCT system, two significant causes of signal disturbance were encountered, namely: electrical noise, which depends on the wall outlet where the OCT system gets its electrical supply, and timing error known as jitter [10], which is caused either by electrical noise and the vibrations of the motor. Jitter is defined as the "time deviation of a phaselocked loop-generated controlled edge from its nominal position" [11], or which simply means that a signal occurs earlier or later than the desired time. These disturbances cause improper capturing of the trigger signal by the oscilloscope and result in falsely widened peaks and lower amplitude of the resulting A-scan after averaging. Because of these, when A-scans are combined to produce a B-scan, the resulting image appears to have 1) blurred edges and 2) misaligned columns of pixels due to jitter, with increased graininess. These are important considerations since our portable setup is designed to be carried and used in different locations. Initial attempts to correct jitter were made by improving the electricals and hardware of the portable OCT system but it was not successful. Hence, a software solution was proposed.

Traditionally, signals are denoised through averaging, and better results are obtained by averaging more signals. This results in a longer signal acquisition time and larger storage space. An advancement in signal processing is through the use of wavelets in denoising signals via thresholding applied in signal denoising [12] and compression [13]. Eventually, wavelet denoising found its way into OCT systems [14].

In Fourier analysis, a function can be represented as a sum of sines and cosines. Similarly, a function can be represented as a sum of scaled and shifted wavelets for wavelet analysis. The main difference between Fourier and wavelet analysis is that wavelets are finite waveforms that are localized in time, in contrast to Fourier transform's since sine and cosine functions extend indefinitely [15]. This makes OCT signal good wavelet transform subjects since the interference patterns obtained in OCT have short coherence length with an envelope localized in time [9].

Wavelet denoising is essentially made up of three processes: The first is breaking down the signal into approximation and detail waveforms using the wavelet transform [16]. The three most commonly used wavelets are Daubechies (Db), Symlets (Sym), and Coiflet (Coif). The next process is eliminating the noise found in the detail waveform based on the selected thresholding method, the most common being soft, hard, and universal thresholding methods. The last step is to reconstruct the signal from the approximation and the remaining detail waveforms using an inverse wavelet transform [17].

Since there are many possible combinations of wavelets, thresholding methods, detail level, and so on, it may become daunting for a researcher to select which particular denoising parameter to choose. It has been shown that in using Haar wavelets, probshrink outperformed older image shrinkage methods in terms of peak signal-to-noise ratio (PSNR) [17]. It was also found out that BayesShrink and Feature-Adaptive
Shrink gave the best mean square error (MSE) and PSNR among the wavelet-based methods that were tested, and good results can also be obtained by combining wavelet-based and spatial filter-based methods. The combination of 'heursure', soft thresholding, and 'sln' scaling has been used to denoise LED LIDAR signals and provided the highest SNR, though there were no significant differences when using other wavelets and thresholding combinations [18].

This study aims to perform signal processing methods, namely, jitter correction and noise reduction through signal averaging and wavelet denoising. This results in an improved OCT signal not just in terms of SNR but of the image quality as well. This is important since it enables us to distinguish actual sample signals from mere noise or image artifacts. As for the B-scan, these signal processing methods will produce a sharp image, allowing us to visualize distinct structures within a sample, thus creating an accurate cross-sectional image of the material under study.

\section{MATERIALS AND METHODS}

\section{A. The Portable TD-OCT System}

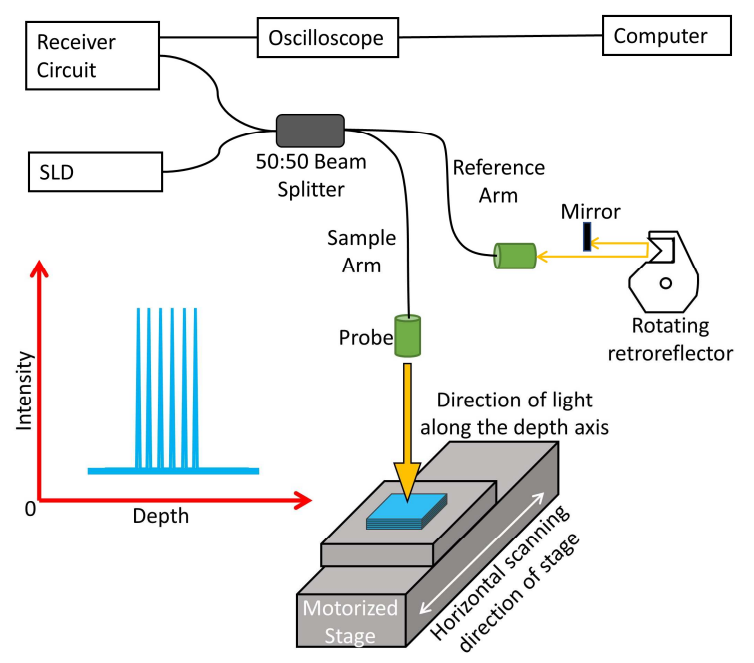

Fig. 1 The experimental setup is showing the A-scan peaks due to the upper and lower surfaces of the glass coverslips.

Figure 1 shows the schematic diagram of our portable OCT system. The specification of our portable TD-OCT system is listed in Table 1. The light source is a superluminescent diode (SLD) with a wavelength of $1310 \mathrm{~nm}$. Incident light from the SLD is split evenly between the reference and sample arms of the 2 × 2 fiber coupler assembly, which acts as the interferometer. The sample and the reference arms of the fiber coupler assembly consists of two collimators, labeled as probe in Fig. 1. Along the sample arm, light exits the probe and penetrates the sample. As light penetrates the sample it encounters reflective and scattering boundaries, and the backscattered light reenters the probe. This backscattered light contains information on the depth and optical properties of these boundaries. Light from the reference arm goes to the optical path scanning mechanism which consists of a rotating retroreflector connected to a motor. The rotating retroreflector directs the light onto a fixed mirror where light is reflected into the probe. This optical path scanning 
mechanism provides a scanning depth of $12 \mathrm{~mm}$ and details can be found in [8].

Backscattered light from the sample and reference arms produced an interference pattern when the sample and reference beams traveled the same optical path length. The interference signal is collected by a photodetector and outputs the interferogram signal and its envelope signal through an electrical filter and an amplifier and digitized by a PicoScope 5444D Mixed Signal Oscilloscope (MSO). Connected to the second channel of the MSO is a photo-interrupter that generates the trigger signal for every full rotation of the retroreflector. The MSO is connected to the laptop's USB port where an A-scan signal is acquired every $0.04 \mathrm{~s}$ using the data acquisition software of the MSO. Aside from the electrical noise, the motor of the rotating retroreflector causes vibration, resulting to jitter in the OCT signals that affects the penetration depth and image quality of the B-scans. To evaluate the performance of the signal processing method developed in this research, a stack of five glass coverslips was used as a sample target. Coverslips were chosen because its thickness is known.

As seen in Fig. 1, the probe on the sample arm is oriented vertically downward into the sample. A single glass coverslip would show two peaks, one for the upper surface and another peak for the lower one. However, a stack of five coverslips only shows six peaks. When the surfaces of two coverslips are in close contact, the two corresponding signal peaks also seem to overlap. The space between the zero marks and the first peak along the x-axis indicates the distance between the probe and the topmost surface of the sample. There are no signals in between the peaks since the material is glass. Hence while the light is passing within the uniformly transparent medium, no backscattering occurs.

The overall flow of the study is presented in Fig. 2. A-scan signals were acquired first using the data acquisition software of the PicoScope before performing the signal processing method. The stack of five coverslips were placed on a motorized translational stage (Sigma Koki SGSP26-100) to be moved horizontally at a regular interval of $2 \mu \mathrm{m}$. The coverslips were moved $2 \mu \mathrm{m}$ horizontally after acquiring 128 A-scans for each sampling point. This process was repeated until 75 sampling points along a straight line on the coverslips were acquired. After the data acquisition, the signal processing method is done, including jitter correction, denoising procedure, and generating a B-scan. A more detailed discussion on the signal processing method is discussed in the next sections.

TABLE I

SPECIFICATIONS OF THE PORTABLE TD-OCT SYSTEM

\begin{tabular}{lc}
\hline Light source & SLD (Anritsu Co. Ltd.) \\
\hline Power & $3 \mathrm{~mW}(\max )$ \\
Center Wavelength & $1310 \mathrm{~nm}$ \\
Spectral Width & $106 \mathrm{~nm}$ \\
Axial Resolution & $7 \mu \mathrm{m}$ \\
Lateral Resolution & $3 \mu \mathrm{m}$ \\
Scanning Rate & $25 \mathrm{scans} / \mathrm{s}$ \\
Scanning Depth & $12 \mathrm{~mm}$ \\
\hline
\end{tabular}

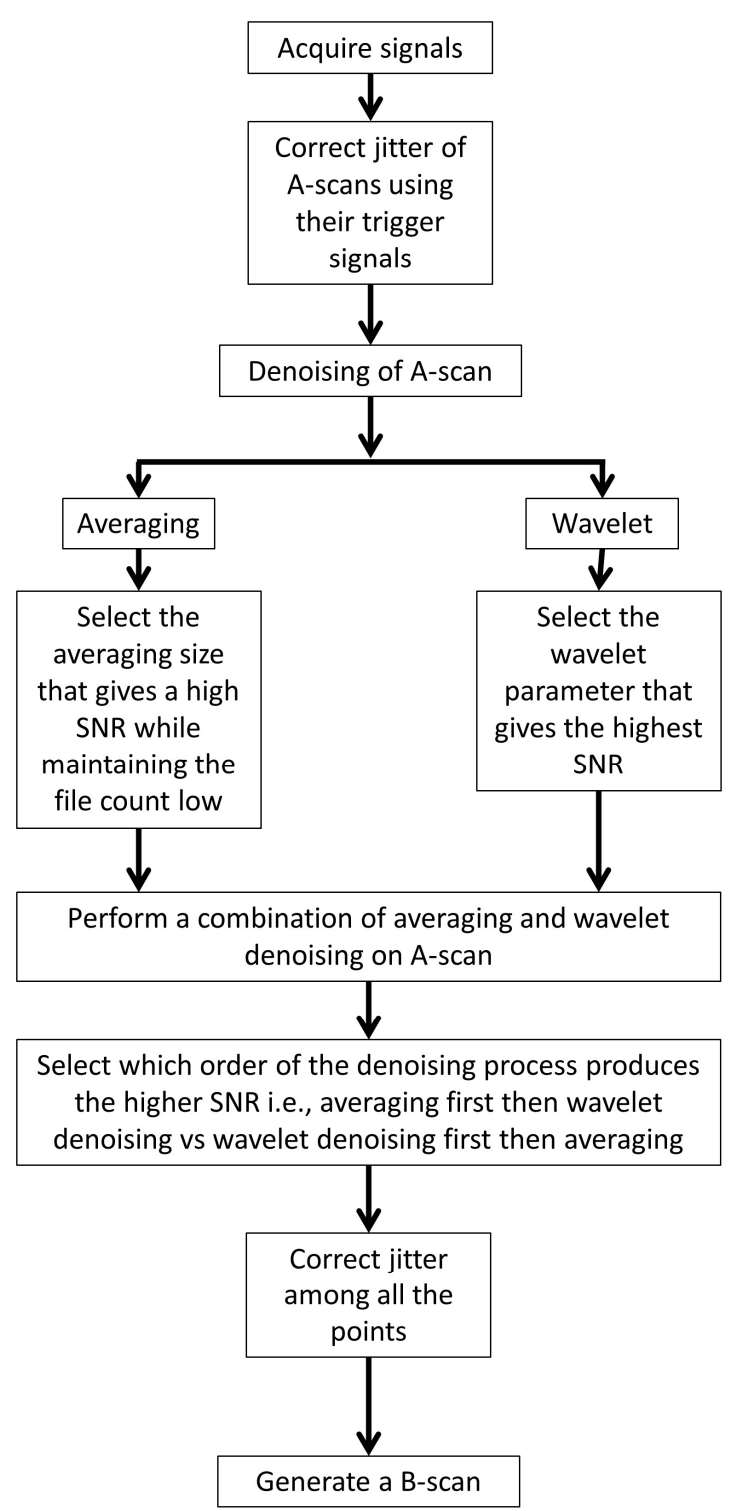

Fig. 2 Overall workflow of the data acquisition and data processing.

\section{B. Jitter Correction}

The first step on the signal processing method of our portable OCT is first correcting jitter. A MATLAB code was written for this process so that each trigger signal has the same time offset along the horizontal axis. To facilitate this, the trigger signals should be noise-free since the downward spikes of the noise might be the ones read by the code instead of the actual downward trend of the trigger. Here, signal averaging cannot be performed since it only fixes random fluctuations of signal amplitudes, i.e., values along the vertical axis and not the variations along the horizontal axis. Also, averaging eliminates time variation among the trigger signals. By visual inspection, the best way to denoise the trigger is to use the smooth function of MATLAB (a moving average filter) with a span of five and then further denoising through the Sym7 wavelet family.

A reference trigger is then selected, and the point in time where the signal's falling edge magnitude is at half-minimum is designated as the reference time, $t_{0}$. The half-minimum value is selected as the basis of assigning $t_{0}$ since, based on our experience, the magnitude of the residual noise does not 
reach this level after denoising. Next, a second trigger signal is selected, and the point in time where its half-minimum is located is designated as $t$. The amount of jitter, $\Delta t$, is obtained by subtracting $\mathrm{t}$ from $\mathrm{t}_{0}$ such that $\Delta t=t_{0}-t$, and the second trigger is shifted left or right depending on the value of $\Delta \mathrm{t}$ so that $t$ becomes equal to $t_{0}$, meaning, the second trigger's falling edge will match that of the reference signal. This process is repeated for the subsequent trigger signals.

The corresponding A-scan of each trigger is then corrected by an amount $\Delta t$. In the case, where a signal has a large value of $\Delta t$ compared to the reference trigger such that it is persistently out of sync even after correction, it is excluded from further processing. After jitter correction, some points will have less than 128 "surviving" A-scans since A-scans and their trigger signals with high jitter amounts were excluded. Thus, for uniformity, for each of the 75 points, only 100 Ascans were utilized for further data processing.

Once jitter is corrected, the A-scans were denoised employing signal averaging and wavelet denoising. The denoising performance was evaluated through the signal-tonoise ratio (SNR) and visual inspection of the resulting waveforms. Ideally, a noiseless signal would be used as the reference. To get a reference signal of each point, the surviving signals are averaged to get the highest possible SNR. Each point then has its own corresponding single reference signal. Once a single, resulting A-scan of each sampling point is produced, jitter among these signals were corrected again to generate a B-scan with sharp edges and correct alignment.

\section{Signal Averaging}

Averaging was performed on each sampling point. Within that sampling point, the SNR values were computed, first for two A-scan signals, then three, and so on until a hundred. This was done for all the points wherein all corresponding SNR's were averaged.

\section{Wavelet Denoising}

Three wavelet families were used, namely Daubechies (Db), Symlet (Sym), and Coiflet (Coif). Signal decomposition was fixed at level 8 . The varying parameters were the wavelet number (3, 5, 7 except for Coiflet, which only had 3 and 5), denoising method (Bayes, Minimax, Stein's Unbiased Risk Estimate or SURE), thresholding rule (soft, hard), and noise estimate (Level-dependent, Level-independent). A single file from a selected point was studied, where all the wavelet denoising method was performed. Afterward, the method which produced the best SNR was compared to the SNR of the averaging method.

\section{RESULTS AND DISCUSSION}

For both denoising methods, this is the computation used for the SNR [17], [19]:

$$
S N R_{d B}=20 * \log _{10}\left(\frac{\sqrt{\sum_{i=1}^{N} S_{D}^{2}}}{\sqrt{\sum_{i=1}^{N} S_{N}^{2}}}\right)
$$

where $S N R_{d B}$ is the SNR in decibels, $N$ is the total number of rows in a signal, $S_{D}$ is the $N$-by- 1 arrays of denoised signals. $S_{N}$ is the $N$-by- 1 arrays of residual noise after denoising, which is also the MSE of $S_{D}$ and reference signal $\left(S_{R}\right)$ such that:

$$
S_{N}=S_{D}-S_{R}
$$

\section{A. Signal Averaging}

A single, raw A-scan signal had an SNR of around 6.31 dB. Figure 3 shows that the SNR can be improved by averaging several signals. The more files averaged, the higher the SNR, with the increasing trend gradually flattening out around 50 A-scans. This means that from 2 files to 50 files, there was an increase of $18.09 \mathrm{~dB}$. While increasing from 50 files to 100 files just improved the SNR by $3.06 \mathrm{~dB}$. This agrees with the observation in signal averaging that, beyond a certain point, further increases in samples may no longer provide much SNR improvement [20].

Producing a high SNR requires several scans per point, and in our oscilloscope settings, $100 \mathrm{CSV}$ files take about 10 seconds and around 66 megabytes of disk storage per point in the sample. Thus, by just using $50 \mathrm{~A}$-scans, we can reduce acquisition time and storage space by at least $50 \%$. This is important, especially when the study involves moving or living subjects wherein long acquisition times are not feasible.

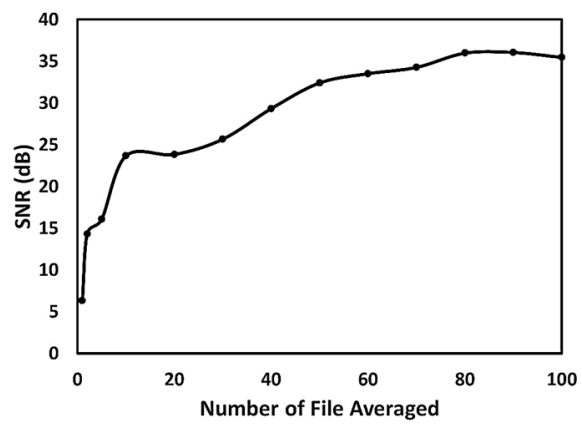

Fig. 3 Improvement in SNR as more files were averaged.

\section{B. Wavelet Denoising}

Table 2 shows the SNR of each combination of parameters for each wavelet family.

TABLE II

\begin{tabular}{|c|c|c|c|c|c|c|c|c|c|c|c|c|}
\hline \multirow[b]{3}{*}{ Thresholding } & \multicolumn{4}{|c|}{ SURE } & \multicolumn{4}{|c|}{ Minimax } & \multicolumn{4}{|c|}{ Bayes } \\
\hline & \multicolumn{2}{|c|}{ Level-Independent } & \multicolumn{2}{|c|}{ Level-Dependent } & \multicolumn{2}{|c|}{ Level-Independent } & \multicolumn{2}{|c|}{ Level-Dependent } & \multicolumn{2}{|c|}{ Level-Independent } & \multicolumn{2}{|c|}{ Level-Dependent } \\
\hline & Soft & Hard & Soft & Hard & Soft & Hard & Soft & Hard & Soft & Hard & Soft & Hard \\
\hline Db3 & 10.26 & 10.75 & 10.26 & 10.30 & 10.26 & 11.35 & 10.26 & 10.60 & 10.26 & 11.25 & 10.26 & 10.49 \\
\hline Db5 & 10.28 & 10.86 & 10.26 & 10.31 & 10.56 & 11.63 & 10.26 & 10.68 & 10.35 & 11.49 & 10.26 & 10.56 \\
\hline $\mathrm{Db} 7$ & 10.37 & 11.02 & 10.26 & 10.33 & 11.16 & 12.01 & 10.31 & 10.79 & 10.54 & 11.85 & 10.27 & 10.66 \\
\hline Sym3 & 10.26 & 10.75 & 10.26 & 10.30 & 10.26 & 11.35 & 10.26 & 10.60 & 10.26 & 11.25 & 10.26 & 10.49 \\
\hline Sym5 & 10.29 & 10.79 & 10.26 & 10.30 & 10.64 & 11.62 & 10.26 & 10.62 & 10.39 & 11.45 & 10.26 & 10.51 \\
\hline Sym7 & 10.31 & 10.81 & 10.26 & 10.30 & 10.82 & 11.70 & 10.27 & 10.61 & 10.43 & 11.46 & 10.26 & 10.49 \\
\hline Coif3 & 10.31 & 10.79 & 10.26 & 10.30 & 10.71 & 11.66 & 10.27 & 10.61 & 10.38 & 11.41 & 10.26 & 10.49 \\
\hline Coif5 & 10.36 & 10.87 & 10.26 & 10.30 & 10.91 & 11.82 & 10.28 & 10.65 & 10.39 & 11.55 & 10.26 & 10.52 \\
\hline
\end{tabular}

THE DiFFERENT SNR VALUES (in dB) OBTAINED USING VARIOUS WAVELET DENOISING PARAmETERS 
The highest SNR values were from $\mathrm{Db} 7$ and Coif 5 with hard thresholding. The lowest values were from Db3 and Sym3 with soft thresholding. The mean SNR of wavelet denoising is $10.63 \mathrm{~dB}$ with standard deviation $0.47 \mathrm{~dB}$. This shows that while Db7 and Coif5 showed the highest SNRs, other wavelet denoising parameters can be nearly as effective. Again, if a single raw file had an SNR of around $6.31 \mathrm{~dB}$, just by performing wavelet denoising, it is possible to improve the SNR two-fold.

In this study, hard thresholding gave slightly better results than soft thresholding. This is explained by the fact that the sample used is a glass coverslip that has sharp and welldefined edges, features that go well with hard thresholding. Furthermore, in soft thresholding, all signals that are not eliminated by denoising are reduced according to the threshold value, which is a feature of this thresholding rule. Thus, it naturally has lower SNR than hard thresholding [17]. It is therefore important to study the properties of the sample in deciding which thresholding is most appropriate.

TABLE III

COMPARISON OF SNRS WHEN USING A COMBINATION OF AVERAGING AND

\begin{tabular}{cccc} 
Number & \multicolumn{3}{c}{ WAVELET DENOISING } \\
\hline $\begin{array}{c}\text { of A- } \\
\text { scans }\end{array}$ & $\begin{array}{c}\text { SNB) } \\
\text { weraging } \\
\text { only }\end{array}$ & $\begin{array}{c}\text { SNB) when } \\
\text { averaging } \\
\text { before } \\
\text { denoising with } \\
\text { Db7 wavelet }\end{array}$ & $\begin{array}{c}\text { SNR (dB) when } \\
\text { denoising with } \\
\text { Db7 wavelet } \\
\text { before } \\
\text { averaging }\end{array}$ \\
\hline 2 & 14.32 & 14.81 & 14.43 \\
5 & 16.09 & 16.71 & 16.14 \\
10 & 23.67 & 25.64 & 23.80 \\
20 & 23.85 & 24.58 & 23.90 \\
30 & 25.67 & 26.23 & 25.71 \\
40 & 29.33 & 30.04 & 29.37 \\
50 & 32.41 & 33.11 & 32.43 \\
60 & 33.52 & 33.68 & 33.52 \\
70 & 34.27 & 34.02 & 34.24 \\
80 & 35.98 & 35.03 & 35.90 \\
90 & 36.06 & 34.73 & 35.94 \\
100 & 35.47 & 34.08 & 35.34 \\
\hline
\end{tabular}

\section{Combination of Averaging and Wavelet Denoising}

Table 3 shows how the SNR increases as more files are used to average. Also, there was a slight improvement observed when performing a combination of averaging and wavelet denoising. There was also no noticeable difference in the order of denoising, whether wavelet denoising was performed first before averaging or vice-versa. Using 50 signals, a slightly higher SNR was obtained by first performing averaging followed by $\mathrm{Db} 7$ wavelet denoising. This has good potential because we can just perform wavelet denoising and reduce the number of acquired A-scans even more, leading to a further reduced acquisition time while still maintaining a high SNR.

\section{B-scan Formation}

B-scans are the cross-sectional images of a sample when several adjacent A-scans are arranged side-by-side. The Bscan's horizontal axis is based on a point's position about the horizontal scanning movement of the probe and its horizontal axis. Its vertical axis is the depth profile of the sample (which is the A-scan's horizontal axis). Lastly, the A-scan's vertical axis values determine the intensity or brightness of a pixel in the B-scan image. The straight lines of the B-scans are the upper and lower surfaces of the glass coverslips when viewed at the cross-section. It is in the B-scan where jitter correction is most appreciated.

Figure 4 is a single raw A-scan of a sampling point, and a set of these adjacent A-scans combines to form a B-scan. Figure 5(a) is a B-scan with jitter, showing jagged, broken lines, which are supposed to be the flat surfaces of the coverslip layers. After jitter correction, Fig. 5(b) shows that each layer is aligned, resulting in smoother lines. Figures 5(a) and (b) appear grainy due to the noise in their A-scans as represented by the tiny peaks in Fig. 4.

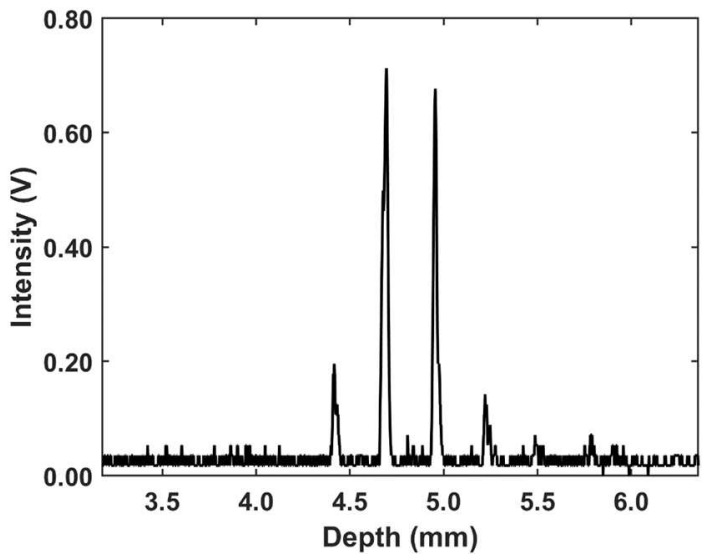

Fig. 4 A single A-scan taken from a point along the horizontal scanning axis.

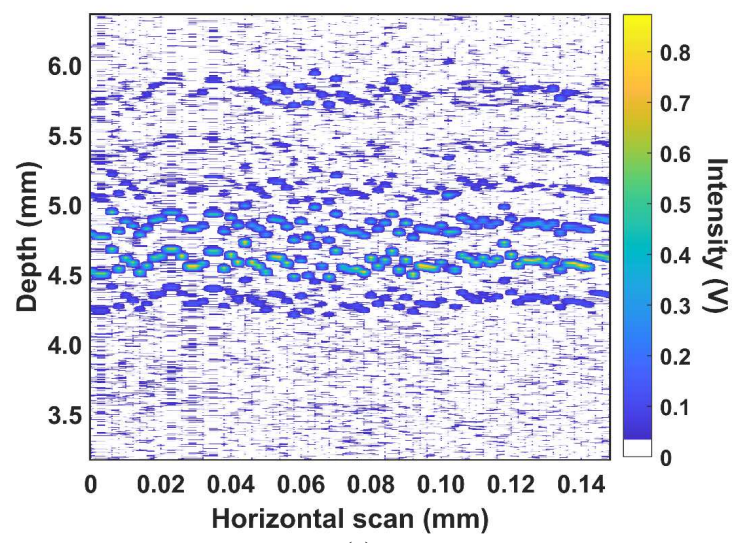

(a)

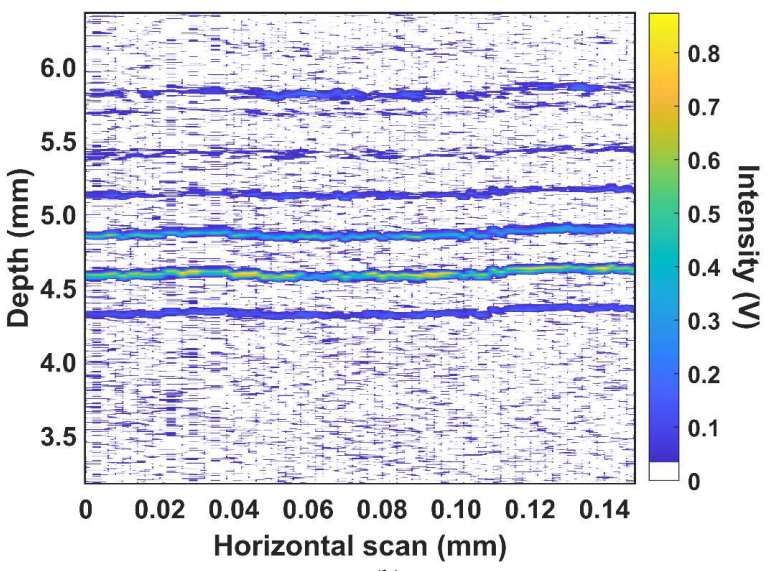

(b)

Fig. 5 B-scans taken using single files from each point scanned horizontally. (a) with jitter, (b) the same signal after correction of jitter. 


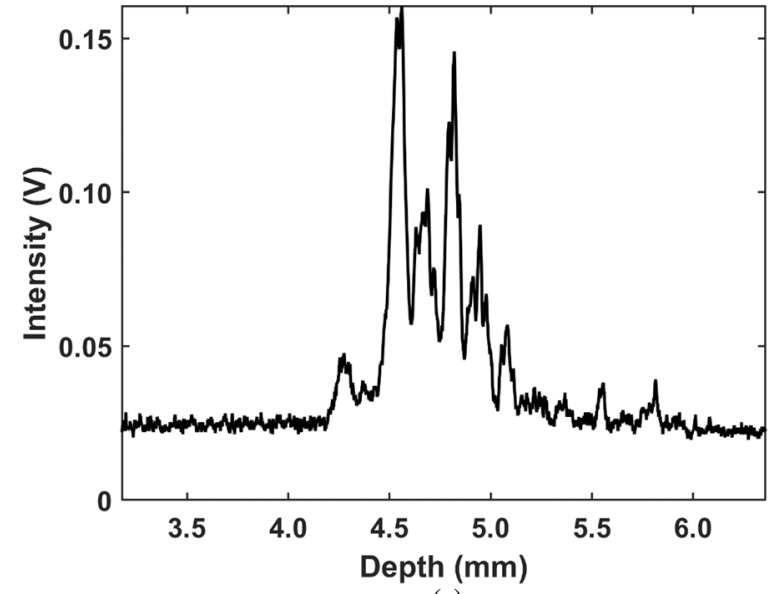

(a)

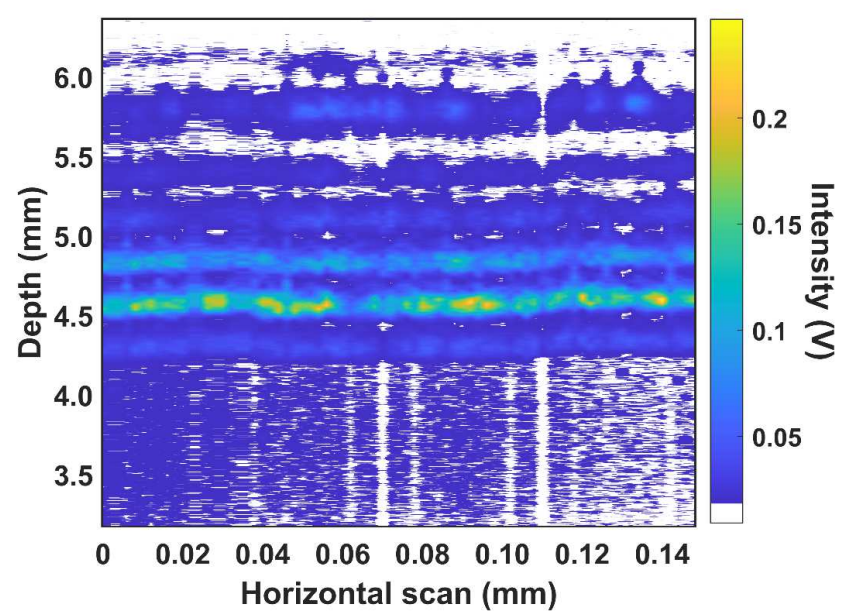

(b)

Fig. 6 Average of $50 \mathrm{~A}$-scans per point when jitter is not corrected. (a) Ascan of a single point (b) B-scan of the sample

It was mentioned earlier that correcting the jitter of the trigger signal is performed first before averaging. Figure 6(a) shows the A-scan when averaging 50 files per point without jitter correction. The depth of the coverslips is not accurately illustrated, which will result in a wrong image produced by the OCT system, as seen in Fig. 6(b), which shows the B-scan when successive A-scans are illustrated on Fig. 6(a) are combined. The lines are jagged, and their wide thickness is due to the broad A-scan peaks. This also results in a drastic reduction of the maximum signal intensity to $\sim 0.15 \mathrm{~V}$ compared to $\sim 0.7 \mathrm{~V}$ in Fig. 4 . This implies that researchers should not rely solely on the SNR when evaluating signal quality.

Figures 7(a) and 7(b) show the result when jitter correction and the best denoising method mentioned in Section III-C was performed using the same set of data. Fifty files were averaged for each of the 75 horizontal scanning points and then performing $\mathrm{Db} 7$ denoising. The surface peaks of the glass coverslips were accurately represented in the A-scan of Fig. 7(a). This produced a continuous straight line representing the layers of the glass, as seen in the B-scan of Fig. 7(b). The removal of the small unwanted peaks produced smoother A-scans, as can be seen in Fig. 7(a), resulting in a sharper and less grainy appearance for the B-scan in Fig. 7(b).

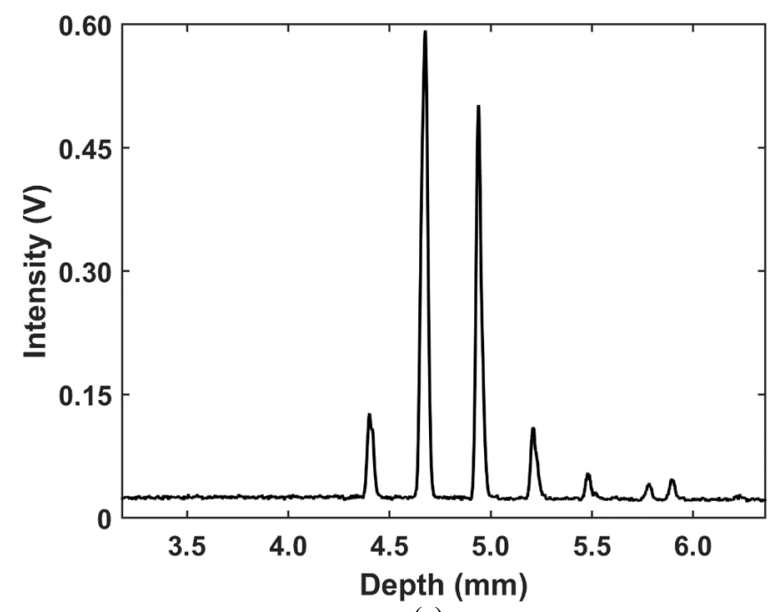

(a)

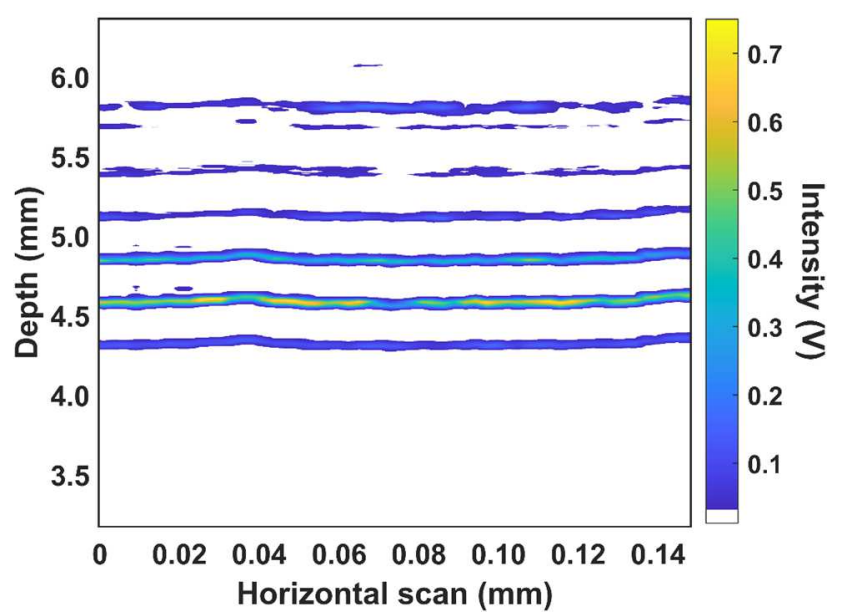

(b)

Fig. 7 (a) A-scan and (b) B-scan when signals were averaged and waveletdenoised after jitter correction.

By using the signal processing method employed in this research, some previously unnoticeable features of the signal can become more visible, just like in the case of Fig. 7(a), wherein a seventh peak around the $6 \mathrm{~mm}$ mark became more prominent. Undistinguishable and imbedded in noise, in the A-scans of Figs. 4 and 6(a), this short peak represents the plate just underneath the glass sample. Thus, proper data processing techniques can help us decide whether a weak signal is from a sample or just part of the noise. We can even extract additional information about the sample for characterization. In this case, each coverslip has an optical thickness of 0.25 $0.26 \mathrm{~mm}$.

\section{IV.CONCLUSION}

To produce accurate A-scans and B-scans on our portable TD-OCT system, jitter correction and signal denoising are necessary. If a hardware solution on the system is not possible, a software solution can be implemented. For jitter correction, wavelet denoising is more appropriate over averaging when processing the trigger signals. In terms of increasing the SNR of our portable OCT system, averaging two or more A-scan signals is superior to wavelet denoising. However, the drawback of averaging is that increasing the number of signals to average requires more time and memory and that further increase of signals produces less benefit. 
Furthermore, before any denoising method is performed, jitter correction should be done first. The order of whether averaging or $\mathrm{Db} 7$ wavelet is performed first does not significantly affect the SNR. Acquisition time and storage space were reduced by at least $50 \%$, and this is important, especially when the study involves moving or living subjects wherein long acquisition times are not feasible. Other wavelet families need to be explored to make full use of their denoising potential. Moreover, using only SNR may not be sufficient when evaluating denoising performance, as visual inspection of the resulting averaged signal is still necessary.

With the jitter correction and signal processing discussed in this study, we improved the A-scan SNR and the B-scan image quality sufficiently. We were able to produce an image showing the five distinct layers of coverslips. Also, producing an accurate image and the optical thickness of the coverslips were obtained. Therefore, it is important to perform jitter correction, signal averaging, and wavelet denoising. This will allow us to properly visualize and characterize other samples using our portable TD-OCT system.

\section{NOMENCLATURE}

$\Delta d \quad$ amount of jitter for the A-scan (mm)

$\Delta t \quad$ amount of jitter for the trigger signal (ms)

$S_{N} \quad$ residual noise after denoising

$S_{D} \quad$ denoised signal

$S N R_{d B} \quad$ SNR in decibels

$t$ the point in time for the trigger's half-minimum value

$t_{0} \quad$ reference time

Coif Coiflet wavelet family

$\mathrm{Db}$ Daubechies wavelet family

MSE mean square error

OCT optical coherence tomography

SNR signal-to-noise ratio

SURE Stein's unbiased risk estimate

Sym Symlet wavelet family

TD time-domain

\section{ACKNOWLEDGMENT}

The authors acknowledge the Commission on Higher Education (CHED) of the Philippine Government for funding the project entitled "Development of a Portable Optical Coherence Tomography System for the Evaluation of Human Skin Analogues". The authors are grateful to the DLSU URCO with project number $47 \mathrm{~F}$ U 2TAY18-2TAY19 and CENSER-DLSU for supporting the project.

\section{REFERENCES}

[1] D. Huang et al., "Optical Coherence Tomography," Science (80-. )., vol. 254, pp. 1178-1180, 1991, doi: 10.1126/science.1957169.

[2] J. Welzel, E. Lankenau, R. Birngruber, and R. Engelhardt, "Optical coherence tomography of the human skin," J. Am. Acad. Dermatol., vol. 37, no. 6, pp. 958-963, 1997, doi: 10.1016/S01909622(97)70072-0.

[3] M. Manfredini et al., "Acne: morphologic and vascular study of lesions and surrounding skin by means of optical coherence tomography," J. Eur. Acad. Dermatology Venereol., vol. 31, no. 9, pp. 1541-1546, Sep. 2017, doi: 10.1111/jdv.14369.

[4] R. Haak et al., "A handheld OCT probe for intraoral diagnosis on teeth," in Optics InfoBase Conference Papers, 2019, vol. Part F142-, doi: 10.1117/12.2527185.

[5] H. Lin, Z. Zhang, D. Markl, J. A. Zeitler, and Y. Shen, "A Review of the Applications of OCT for Analysing Pharmaceutical Film Coatings," Appl. Sci., vol. 8, no. 12, p. 2700, 2018, doi: 10.3390/app8122700.

[6] V. Srivastava, D. Dalal, A. Kumar, S. Prakash, and K. Dalal, "In vivo automated quantification of quality of apples during storage using optical coherence tomography images," Laser Phys., vol. 28, no. 6, Jun. 2018, doi: 10.1088/1555-6611/aab485

[7] F. Atry, I. J. De La Rosa, K. R. Rarick, and R. Pashaie, "Design and Implementation Guidelines for a Modular Spectral-Domain Optical Coherence Tomography Scanner," Int. J. Opt., vol. 2018, pp. 1-22, 2018, doi: 10.1155/2018/3726207.

[8] T. Shiina, Y. Moritani, M. Ito, and Y. Okamura, "Long-optical-path scanning mechanism for optical coherence tomography," Appl. Opt., vol. 42, no. 19 , pp. 3795-3799, 2003, doi: 10.1364/ao.42.003795.

[9] W. Drexler and J. G. Fujimoto, Optical coherence tomography: Technology and applications, second edition. Springer International Publishing, 2015.

[10] S. Davidson, "Instrumentation: Measuring digital clock stability and jitter with an oscilloscope," in EE: Evaluation Engineering, vol. 57, no. 11, Nelson Publishing Inc., 2018, pp. 20-21.

[11] Joint Electron Device Engineering Council, "JEDEC Standard Definition of Skew Specifications for Standard Logic Devices JESD65B," no. September, Arlington, VA: JEDEC Solid State Technology Association, 2003, p. 1.

[12] R. Guhathakurta, "Denoising of image: A wavelet based approach," in 2017 8th Industrial Automation and Electromechanical Engineering Conference, IEMECON 2017, Oct. 2017, pp. 194-197, doi: 10.1109/IEMECON.2017.8079587.

[13] M. A. Gungor and K. Gencol, "Developing a Compression Procedure Based on the Wavelet Denoising and JPEG2000 Compression", Optik (Stuttg)., vol. 218, p. 164933, Sep. 2020, doi: 10.1016/j.ijleo.2020.164933.

[14] T. Suzuki, B. Liu, and S. Choi, "3D thickness measurement using pulse-driven optical coherence tomography based on wavelet transform," in Proceedings of SPIE - The International Society for Optical Engineering, Sep. 2019, p. 45, doi: 10.1117/12.2534005.

[15] J. Burgess, Wavelets: Principles, analysis and applications. Nova Science Publishers, Inc., 2018.

[16] R. Gonzalez and R. Woods, Digital Image Processing. New York: Pearson Education, Inc., 2018.

[17] W. Van Drongelen, Signal processing for neuroscientists. Elsevier, 2018.

[18] P. M. Ong, M. C. Galvez, E. Vallar, and T. Shiina, "Wavelet denoising applied to light emitting diode lidar signal," Adv. Sci. Lett., vol. 23, no. 3, pp. 1374-1378, Mar. 2017, doi: 10.1166/as1.2017.8352.

[19] M. Misiti, Y. Misiti, G. Oppenheim, and J.-M. Poggi, Wavelet toolbox: User's Guide, Version 5. Natick MA: Mathworks Inc., 2020.

[20] H. Mark and J. Workman, "Calibration Transfer - The Instrumentation Aspects," in Chemometrics in Spectroscopy, 2nd ed., H. Mark and J. Workman, Eds. Academic Press, 2018. 